\title{
CRISIS DE LA TRANSMISIÓN Y FIEBRE DE LA INNOVACIÓN ${ }^{1}$
}

\section{Transmission Crisis and Innovation Fever}

François-Xavier BELLAMY

Asociación Philia

https://www.philia-asso.fr/

Fecha de recepción: 20/11/2020

Fecha de aceptación: 27/01/2021

Fecha de publicación en línea: 01/03/2021

Cómo citar este artículo: Bellamy, F.-X. (2021). Crisis de la transmisión y fiebre de la innovación. Teoría de la Educación. Revista Interuniversitaria, 33(2), 169-178. https:// doi.org/10.14201/teri.25407

\section{RESUMEN}

Este artículo propone destacar las potencialidades educativas propias de la escuela vivida como una comunidad educativa. Toda escuela debería asumir el sentido más profundo de su etimología griega. La tesis principal del texto es que no hay destino para nosotros, no hay novedad, no hay innovación, no hay verdadero cambio, no hay libertad, sin recibir primero de la historia, a veces de la historia más antigua, aquello que puede alimentar nuestra capacidad de inventar y de crear. Esta es la razón por la que, en realidad, la misma oposición entre el pasado y el futuro no puede tomarse en serio.

Palabras clave: Bellamy; tradición; trasmisión; herencia; permanencia; cambio; humanismo.

1. Este texto es la transcripción de la conversación pública mantenida, vía internet, con el profesor Bellamy el 20 de noviembre de 2020 dentro de la actividad "Diálogos sobre educación" organizada por la revista Teoría de Educación. Revista Interuniversitaria. Agradecemos al profesor Bellamy su generosidad para participar en esta conversación. Agradecemos a la profesora Tania Alonso-Sainz la organización de este evento y a la profesora Bianca Thoilliez haber realizado la traducción simultánea. Transcripción: Laura Suárez Gil. Disponible en: https://revistas.usal.es/index.php/1130-3743/dialogos 


\section{ABSTRACT}

This article proposes to highlight the educational potentialities of the school lived as an educational community. Every school should assume the deepest meaning of its Greek etymology. The main thesis of the text is that there is no destiny for us, there is no novelty, there is no innovation, there is no true change, there is no freedom, without first receiving from history, sometimes from the oldest history, what can feed our ability to invent and create. This is the reason why, actually, the very opposition between the past and the future cannot be taken seriously.

Key words: Bellamy; tradition; transmission; heritage; permanence; change; humanism.

Buenas tardes, estoy muy feliz de poder participar y compartir esta tarde con vosotros este momento de intercambio, a pesar de la distancia. Es un gran honor para mí poder encontraros y espero que podamos alargar esta conversación con un encuentro más real cuando las circunstancias lo permitan. Me siento muy honrado de haber recibido esta invitación y, querida Tania, muchas gracias por tus palabras de presentación con las que podemos iniciar este intercambio. Estoy muy contento de que Los desheredados y Permanecer haya podido acompañar vuestras reflexiones ${ }^{2}$.

Querría hoy, muy modestamente, volver sobre la intuición que traté de desarrollar en estos dos textos. He recibido un cierto número de preguntas que me habéis compartido. Lamento no poder responder en vuestra bella lengua y perdón por tener que hacerlo en francés. Agradezco mucho a Bianca Thoilliez que haya aceptado a hacer este trabajo de interpretación.

\section{El Milagro de LA LIBERTAD}

En las cuestiones que habéis planteado, uno de vosotros escribía "¿ies necesario preferir el futuro o el pasado?» y me parece que el trabajo que he intentado hacer tanto en Los desheredados como en Permanecer, consiste en demostrar que, al contrario de lo que la modernidad querría, estas dos dimensiones de la existencia no pueden darse de manera opuesta. En Los desheredados buscaba entender por qué durante toda mi formación como profesor había escuchado decir que no había que transmitir una herencia, que había que dejar a los estudiantes inventar su propio futuro. Como si, en el fondo, escribir nuestro destino futuro consistiese necesariamente en deshacerse de nuestro pasado.

2. F.-X. Bellamy, Los desheredados. Por qué es urgente transmitir la cultura. Encuentro, 2018. Permanecer. Para escapar del tiempo del movimiento perpetuo. Encuentro, 2020. 
En realidad, creo que es, exactamente, todo lo contrario. Cuando hablo ahora, en este momento, cuando hablamos, cuando pensamos también, lo hacemos utilizando palabras que nos vienen a veces de la historia más antigua. Las primeras palabras que hemos aprendido a pronunciar, la palabra 'papá' o 'mamá', que son prácticamente comunes en nuestras dos lenguas, tienen raíces que se remontan hasta el indoeuropeo. Esas palabras tienen, por lo tanto, varios miles de años de historia.

Cuando hemos aprendido a hablar con esas palabras, cuando hemos aprendido a pensar con esas palabras, no se nos ha impedido inventar nuestra propia libertad. Muy al contrario, nunca habríamos podido pensar libremente sin esas palabras que hemos recibido. Y quienes nos las han transmitido tampoco podrían predecir qué íbamos a hacer con la lengua que aprendíamos. Con esas palabras podemos hacer el bien y podemos hacer el mal. Estamos abiertos por esas palabras al vértigo de la libertad. Podemos utilizar la lengua en una diversidad infinita de usos. Y el milagro de la libertad, el milagro de la educación es que una libertad nueva nace porque es alimentada, nutrida, fecundada, por esta herencia del pasado.

No hay destino para nosotros, no hay novedad, no hay innovación, no hay verdadero cambio, no hay libertad, sin recibir primero de la historia, a veces de la historia más antigua, aquello que puede alimentar nuestra capacidad de inventar y de crear. Esta es la razón por la que en realidad creo, en el fondo, que esta misma oposición entre el pasado y el futuro no puede tomarse en serio. Políticamente no existen, por un lado, los progresistas, que sería el campo del futuro, contra los conservadores, que sería el campo del pasado. Una oposición así simplemente esta desprovista de toda significación.

Y lo que me ha parecido esencial en la crisis que atravesamos, es que nace precisamente de esta ficción. Nace de la idea que nos hacemos, de que podemos inventar nuestra libertad de mañana a cambio de nuestra herencia y del sacrificio de nuestra historia, de nuestro pasado, de nuestras raíces, de nuestros principios, de todo aquello que permanece en el fondo, de aquello que queda idéntico en el interior de una sociedad.

Hannah Arendt en La condición del hombre moderno escribía que la educación es esencialmente conservadora. Pero para ella esto no es una forma de decir que la educación debe de encerrarnos en la repetición del pasado, en la repetición de lo mismo. Lo que Hannah Arendt quiere decir es que el milagro de la libertad, que nos hace posible introducir una novedad en la historia, a través del juego de la acción; el milagro de la libertad por el cual inauguramos algo nuevo, no podría nacer si no se fundase en la recepción de una herencia a través del trabajo de la educación. Y es así como la educación, es conservadora y al mismo tiempo la única ocasión posible para una renovación del mundo. La única manera de ofrecer al mundo lo que Hannah Arendt llamaba la natalidad, es decir, la novedad de cada generación, que aporta a lo real su capacidad de inventar. 
Para poder escribir el futuro, escribe Hannah Arendt, debemos inspirarnos en la única cosa de la que disponemos, dado que el futuro no nos da nada puesto que todavía no existe. El futuro no nos aporta nada, somos nosotros quienes debemos aportarle y para poder darle algo debemos primero recibir, y lo que podemos recibir viene de quienes nos preceden, de aquello que permanece. Al escribir Permanecer no buscaba afirmar que habría, por un lado, el campo del movimiento, del cambio y de la libertad y, por otro lado, el partido del inmovilismo y de la estabilidad.

El debate entre Parménides y Heráclito ha estructurado toda la civilización occidental. Pero si lo miramos bien, veremos que la única cosa que puede salvar al movimiento es la existencia de una permanencia. Si nada es inmóvil, entonces nada puede permitir percibir el movimiento. Y eso es así también en la misma dimensión física de este término.

Heráclito, que encarna la filosofía del cambio, afirma que todo fluye, que nunca nos bañamos dos veces en el mismo río. Pero para ver que el río fluye, hace falta estar cerca del río y mirarlo pasar. Si todo es líquido, si todo es móvil, entonces el mismo movimiento resulta imposible, imperceptible y no puede ser salvado. No puedo haber progreso sin la condición de que el progreso esté orientado hacia el mundo estable que le dé su significación.

Cuando nos comprometemos en el campo político no debemos buscar solamente estar a la última, estar a la moda, a seguir la tendencia del momento. Lo que da sentido al esfuerzo político para el progreso es la búsqueda de un ideal que no varía con el tiempo. Es la búsqueda del bien, de la justicia, y esta es una búsqueda eterna y podemos progresar si los cambios de nuestra época nos acercan a esos objetivos. No porque algo sea nuevo es necesariamente mejor.

Así, sobre las cuestiones que me planteabais y sobre las que podemos volver ahora, creo que hay una llamada a superar las contradicciones que demasiado a menudo nos trampean, tanto en el trabajo pedagógico como en el compromiso político. Y estos dos terrenos hacen eco en la experiencia que vivo. Me parece que no buscamos, no debemos buscar, fijar nuestro mundo en un pasado idealizado, como tampoco deberíamos mostrar resentimiento hacia la realidad buscando sistemáticamente cambiarlo todo, transformarlo todo.

Lo que da sentido al trabajo educativo es la experiencia maravillosa de suscitar en nuestros estudiantes una libertad nueva de la que no podemos predecir los frutos, y que no podremos medir, permitiendo que sus espíritus se vean aumentados por el encuentro con aquello que hemos heredado.

Lo que da sentido a la política es buscar apoyarnos sobre esta sabiduría, el punto fijo que orienta nuestro esfuerzo, para permitir un progreso auténtico.

Esta superación del enfrentamiento entre el pasado y el futuro, que yo he tratado de abordar en estos dos libros y que estoy muy contento esta tarde de tener la suerte y el privilegio de poder evocar con vosotros, son cuestiones que creo 
cruciales para superar la crisis de la modernidad que vivimos y que atravesamos hoy juntos, más allá de la frontera entre nuestros dos países, en el destino común de la civilización europea.

\section{HACER FRENTE A LA REALIDAD CON LUCIDEZ Y ESPERANZA}

En el fondo no soy pesimista. Un gran escritor francés, Georges Bernanos, muy cercano a España, escribía que el optimismo y el pesimismo son las dos caras de un mismo error, que "El optimista es un imbécil feliz, el pesimista es un imbécil infeliz». Me parece que lo que cuenta es hacer frente a la realidad con lucidez para poder encontrar la fuerza de reaccionar con esta virtud esencial que es la esperanza. La esperanza es totalmente distinta al optimismo, supone aceptar la dificultad del momento que atravesamos, dado que si no hay dificultades no hay necesidad de esperanza. Pero la esperanza es la apuesta de que, a pesar de las dificultades, podemos encontrar otra aurora, dice Bernanos, podemos avanzar hacia otro día y es lo que creo que está en el corazón del trabajo educativo. Todo educador auténtico se encuentra animado por la esperanza.

Hoy no soy más optimista que hace algunos años, no hay realmente muchas razones para serlo, vemos que nuestras sociedades se encuentran profundamente fracturadas. En Francia, en particular, la educación se ha visto directamente afectada por el atentado contra un profesor recientemente decapitado a causa de una de sus clases. No hay muchas razones para ser optimista, y, sin embargo, sigo estando convencido de que, si somos capaces, de nuevo, de transmitir lo que hemos recibido, encontraremos en nuestros estudiantes la sed infinita de aprender. Y es esta esperanza la que anima, lo veo a mí alrededor entre muchos de quienes se embarcan en la misión educativa, la preocupación de preparar un mundo mejor para el futuro.

\section{Multiculturalismo, PlURAlismo y BIEN COMÚN}

Me parece que debemos distinguir entre pluralismo y multiculturalismo. Una sociedad siempre estará hecha de la pluralidad de las personas que la constituyen, de la riqueza que representan la singularidad de nuestras representaciones del mundo, de nuestras maneras de percibirlo y de acercarnos a él. Y es justamente la experiencia extraordinaria de la educación, que cuando das la misma clase a 30 estudiantes, cada uno saca cosas diferentes. Cuando transmites la misma lengua a niños, dirán cosas diferentes con las mismas palabras aprendidas. Esta es la riqueza de una sociedad. Aristóteles escribió en Ética a Nicómaco que todo hombre tiene algo de particular que aportar a la verdad, y creo que es una de las afirmaciones filosóficas más bellas: todo hombre tiene algo particular que aportar a la verdad. No se trata, por lo tanto, en ningún caso, de ser relativista pues nos podemos aproximar a la realidad desde miradas variadas que no son, necesariamente, contradictorias. 
Cuando surge una contradicción, lo cual forma parte de la vida de toda sociedad, puede suscitar un diálogo, una conversación. Para ello es necesario, sin embargo, que haya una lengua común, hace falta también que haya una cultura común y el pluralismo en una sociedad no puede vivirse más que cuando tenemos en común algo que nos reúna.

Vivimos hoy el fracaso de un modelo multicultural, ese modelo que ha visto comunidades yuxtaponerse unas a otras, alejarse cada vez más unas de otras, sin reconocerse vínculos. El trabajo de la educación tiene justamente este elemento de decisivo: al mismo tiempo que hace crecer la libertad de aquellos que aprenden, también suscita la conciencia de ese vínculo común. No queremos una autoridad que viniese a uniformar, que tratase de repetir en moldes la vida, que buscase que todos nos adaptásemos a un mismo modelo, a hacernos a todos iguales porque, gracias a la transmisión de una herencia y a la autoridad de los autores que podemos leer, descubrimos lo que alimenta nuestra libertad, al mismo tiempo que se funda en nosotros el sentido de ese vínculo común. Esta es la razón por la que creo que el modelo del multiculturalismo que hemos querido imponer a nuestras sociedades, no tiene futuro. Hay, evidentemente, un lugar, en nuestros países, en nuestras sociedades, en nuestras culturas, para la libertad, para la singularidad de cada persona con su historia, con sus talentos y su sensibilidad. Pero lo que hace de esta singularidad un enriquecimiento es la posibilidad del diálogo, que viene de una cultura común.

\section{CRECER lejos de las nUevas tecnologías}

Me parece que la clave para preparar el mundo del mañana y para preparar a los niños a ese mundo de las nuevas tecnologías consiste, precisamente, en permitirles crecer lejos y apartados de esas tecnologías; y asegurar para ellos una distancia que permite la libertad. De hecho, si lo miramos bien, aquellos que han inventado las nuevas tecnologías no habían aprendido en la escuela el código informático o los fundamentos de la industria numérica. Quienes inventaron Google o Apple no habían sido primero educados en esas técnicas contemporáneas. Al contrario, habían estudiado Matemáticas - aprendiendo de ellas conocimiento con una antigüedad de miles de años- lo que les permitió desarrollar una capacidad intelectual para inventar algo nuevo. Del mismo modo, cuando Steve Jobs desarrolló Apple, siempre contó que el sentido del diseño, del grafismo tan particular de sus productos, le había venido por el aprendizaje de la caligrafía finesa, un arte con más de seis mil años de historia. Sería un error creer que para que nuestros alumnos sean competitivos mañana en el mundo digital, hay que enseñarles el código informático en el colegio. Les cambiará diez veces antes de que puedan ser empleados en el mercado del trabajo.

Al contrario, debemos estructurar en ellos el espíritu lógico, la capacidad de análisis, debemos construir en sus espíritus una capacidad de reflexión que 
mañana hará de ellos personas creativas e imaginativas, incluido en el ámbito de las nuevas tecnologías. Lo sabemos bien, los niños no necesitan que les enseñemos a utilizar un smartphone, y de hecho el smartphone se compra y se adquiere sin instrucciones de uso, no hace falta tener una competencia particular, actualmente, es una experiencia universal. Cuando los adultos pretenden enseñar a los niños a utilizar un smartphone o un ordenador, se dan cuenta de que a menudo son los niños quienes saben más que ellos. Lo propio de la revolución digital es que son a menudo los jóvenes quienes enseñan a los más viejos, lo cual es totalmente inédito en la historia de la técnica. Los niños no necesitan que se les enseñen a usar un smartphone, se sienten espontáneamente inclinados hacia el universo de lo digital.

En cambio, el mundo en el que vivimos es uno en el que tienen más necesidad que nunca de que la escuela les permita reencontrarse con ese objeto decisivo para hacer nacer en ellos la libertad y que se llama el 'libro'. Cuando empiezas a hacer una investigación en Google, ya sabe uno lo que quiere encontrar y el objetivo es ir lo más rápido posible al resultado de la búsqueda. El mundo de lo numérico es el mundo de la inmediatez, el mundo de la aceleración. Hemos paso de la $2 G$, a la $3 G$, a la $4 G$ y a la $5 G$, y tenemos teléfonos y ordenadores cada vez más eficientes para ir cada vez más y más deprisa, de la pregunta a la respuesta y del deseo a su consumación. La lógica de lo digital, la lógica de la inmediatez es exactamente al revés de la lógica de la lectura y es, sin duda, la razón por la que el mundo de los teléfonos ha hecho tan difícil para nosotros la paciencia del libro. Cuando uno abre un libro, por definición, uno no sabe lo que se va a encontrar y acepta seguir un camino que todavía no conoce. Lo que cuenta no es llegar lo antes posible a la última página, sino el tiempo mismo de la lectura del libro, su espesor, su sustancia. El encuentro con un autor puede así aumentarte, puede llevarte a conocer cosas que no conocías, cosas que ni siquiera pensabas desear. La palabra 'autor' viene del latín auctor que, a su vez, viene del verbo aumentar. El libro aumenta mi libertad, me aumenta a mí mismo. A diferencia de lo digital, que es un juego de círculo cerrado, el libro es una experiencia que me conduce a encontrar aquello que no buscaba. Así, la paciencia del libro despierta la libertad y es más necesaria que nunca en el mundo de las nuevas tecnologías.

Por supuesto, los niños de hoy vivirán en un mundo numérico, pero me parece que para prepararlos, para garantizar su libertad frente a estas herramientas, para garantizar que mañana no estemos poseídos por aquello que poseemos, para que mañana no estemos dominados por nuestras propias herramientas, tenemos que poder madurar esta libertad que el libro puede darnos. La palabra libro viene del latín liber y su etimología misma remite a la libertad y creo que la Pedagogía más adaptada al mundo contemporáneo es aquella que, justamente, sabrá apartarse de las últimas novedades para volver a esta fuente de libertad interior. Heidegger escribió «aquellos que no saben envejecer molestan siempre a los jóvenes con las últimas novedades, los que saben envejecer devuelven a los jóvenes a lo esencial» y creo que es una buena máxima para la Pedagogía de hoy. 


\section{ESCUELAS PÚBLICAS Y EDUCACIÓN DENSA}

Soy profesor en la enseñanza pública. He sido profesor durante más de 10 años y me parece que la libertad de enseñanza es por supuesto fundamental en democracia. Soy un antiguo alumno de la enseñanza privada y creo que la libertad de enseñar debe ser defendida absolutamente. Pero creo que también, como profesor, debemos preocuparnos de todos los alumnos y poder acompañar a los niños, con independencia del lugar donde estudien.

A pesar de que solo soy un profesor de Filosofía, me parece que la crisis que vivimos hoy tiene de manera innegable una dimensión espiritual. No es solo una crisis de la Pedagogía. Charles Péguy, en El dinero, escribió «que una sociedad que no sabe enseñar es una sociedad que no sabe enseñarse a sí misma». Las crisis de la enseñanza, escribía él, son crisis de la vida. Las crisis de la enseñanza son crisis de la vida. La crisis educativa actual, en mi país en particular, no es solo un problema técnico, pedagógico o social, es una crisis de la vida y es una crisis espiritual. Pero tenemos que poder tratar de responder en todos los lugares donde se desarrolle un trabajo educativo y la enseñanza pública forma parte, por su puesto, tanto como la enseñanza privada. Me parece que sería demasiado triste apartar de nuestras preocupaciones todo un sector de la enseñanza donde se vive ya un trabajo de transmisión, allí donde la llama de la vinculación a este esfuerzo educativo está todavía viva y es alimentada.

\section{LA CAPACIDAD DE MARAVILLARSE}

No es en absoluto una cuestión sencilla preguntarse por el modo de llevar a cabo, en el contexto actual, la formación ideal de un futuro profesor. Si hay algo todavía más difícil que formar estudiantes es formar a profesores. Y no pretendo poder daros un método, un camino a seguir o una especie de instrucciones de uso. Pero diría, simplemente, que el mejor modo de buscar ser un buen profesor es seguir toda la vida siendo un estudiante. Lo que es, sin duda, más esencial para un profesor es seguir aprendiendo, seguir descubriendo, mantener viva la curiosidad y preservar la capacidad de maravillarse. Si nuestros estudiantes nos escuchan, si les pedimos silencio, no es porque seamos genios ni más inteligentes que ellos, sino porque estamos tan maravillados por aquello que hemos aprendido que queremos compartirlos con ellos y que lo puedan, a su vez, escucharlo. Y, desde este punto de vista, el mejor profesor es aquel que todavía sigue estando lo bastante animado por la búsqueda de la verdad que continúa siempre aprendiendo. No es un ejercicio fácil porque el trabajo de la enseñanza es un esfuerzo exigente, a menudo árido, y en el que es muy fácil ponerse a repetir, a volver a decir, otra vez, siempre lo que ya uno sabe. Es muy difícil volver a ponerse en camino. Es muy exigente no contentarse con volver a hacer lo que ya hemos hecho, con lo que ya sabemos. En el fondo, el principio fundamental de la filosofía — que es el de reconocer que la 
verdad está siempre más allá de aquello que podemos decir y que la búsqueda será el camino de la existencia-, es también la mejor manera de continuar formándose, no para ser un día el profesor perfecto y completo, sino para buscar convertirse siempre en el mejor profesor posible, siendo siempre un alumno y dejándose elevar por aquello que seguimos aprendiendo.

\section{LA URGENCIA DE LA TRANSMISIÓN}

No querría entrar en una discusión sobre la enseñanza en Francia. Creo que la última reforma me parece reprochable porque sigue disminuyendo el número de horas de enseñanza en las asignaturas dedicadas a la cultura general y a las humanidades. Francia tenía hasta ese momento un programa escolar específico de 3 itinerarios dentro de la enseñanza general, así como la enseñanza técnica y la enseñanza profesional, y en lugar de esos itinerarios, que aseguraban una coherencia y una enseñanza completa, vemos hoy multiplicarse un liceo hecho de opciones a la carta, al que le falta la unidad de esta enseñanza general. Yo creo que cedemos a una forma de fascinación hacia el modelo anglosajón que es el de la especialización. El modelo de la especialización precoz que consiste en renunciar a la idea continental de la cultura general. Blaise Pascal, el filósofo, escribió «es mucho más bello saber un poco de todo que saber todo sobre una cosa». Después las revoluciones pedagógicas, me parece que renunciamos a esta idea, que preferimos un modelo anglosajón, americano, orientado hacia la competición económica para hacer alumnos que estén muy preparados en un solo ámbito y creo que perderemos mucho, desgraciadamente, en el plano del desarrollo humano, intelectual y espiritual, que permitía el enraizamiento en esta cultura general.

Es un tema preocupante, y terminaré con esto. Una sociedad puede cometer muchos errores que pueden ser reversibles. Si has sido demasiado laxo en materia de seguridad, un día puedes volver a poner policías en las calles y restablecer el orden. Si has gastado demasiado dinero público, un día puedes volver a ahorrar y reorganizar las cuentas. Pero si has fallado en el trabajo de la enseñanza, habrá saberes que no se han transmitido y, ¿quién podrá enseñarlos? La enseñanza es, sin duda, el único ámbito donde podemos cometer errores definitivos. Si hemos dejado de transmitir determinados saberes, por ejemplo, en Francia, el Latín y el Griego, lenguas antiguas que son tan fundamentales para entender el mundo y nuestra propia vida interior, si hemos dejado de transmitirlas, ¿quién podrá dar testimonio de que son fecundas para el espíritu? Con los tesoros que no transmitimos, perdemos hasta la conciencia de que son tesoros. Y no digo esto para parecer pesimista, sino solo para que tomemos conciencia de que la misión de la enseñanza se encuentra hoy marcada por una forma de urgencia esencial, esta urgencia de transmitir que es, creo, el mayor desafío político de hoy. 
Quiero decir de corazón a todos aquellos que enseñáis, a todos aquellos que contribuís a esta maravillosa empresa de la enseñanza — que yo he dejado unos años, ahora estoy en el Parlamento Europeo, pero espero regresar pronto-, a todos aquellos que compartís esta bella misión, mi más profundo reconocimiento por el trabajo esencial que hacéis, por hacer nacer en los niños a los que acompañáis, toda la libertad y creatividad de la que el mundo de mañana necesitará. 